\title{
Takotsubo Syndrome a Rare Entity in COVID-19: a Systemic Review-Focus on Biomarkers, Imaging, Treatment, and Outcome
}

\author{
Kamal Sharma $^{1} \cdot$ Hardik D. Desai $^{2}$ (D) $\cdot$ Jaimini V. Patoliya ${ }^{3} \cdot$ Dhigishaba M. Jadeja $^{4} \cdot$ Dhruv Gadhiya $^{5}$
}

Accepted: 5 January 2021 / Published online: 11 January 2021

(C) The Author(s), under exclusive licence to Springer Nature Switzerland AG part of Springer Nature 2021

\begin{abstract}
Takotsubo syndrome(TTS) is attributed to catecholamine surge, which is also observed in COVID-19 disease due to the cytokine storm. We performed a systematic literature search using PubMed, Embase, and the Cochrane Central Register of Controlled Trials retrospectively to identify COVID-19-associated TTS case reports and evaluated patient-level demographics, laboratory markers clinical attributes, treatment given, and outcomes. There are 27 cases reported of TTS associated with COVID-19 infection of which $44.5 \%$ were male. Reported median age was 57 years (IQR: 39-65) and 62.95 years (IQR: 50.5-73.5) in case series and individual patients' cases in database, respectively. The time interval from the symptom onset to TTS diagnosis was median 6.5 days (IQR: 1.0-8.0) in case series and 6.7 days (IQR: 4-10) in individual patients' database. The median LVEF was 36\% (IQR: 35-37) and 38.15\%(IQR: 30-42.5\%-[male: 40.33\% (IQR: 33-44.2)] and female [37.15\% (IQR: 30-40)] in case series and individual-patients' database, respectively. Troponin was elevated in all patients except one patient. $77.2 \%$ patients of TTS with COVID-19 had an elevated C-reactive protein and/or D-dimer. Twelve out of 22 (54.5\%) patients developed cardiac complication such as cardiogenic-shock, atrial fibrillation, acute heart failure, supraventricular tachycardia, and biventricular heart failure. Nineteen out of $26(73.07 \%)$ patients were discharged, and three were hospitalized due to acute respiratory distress syndrome and needed extracorporeal membrane oxygenation or ongoing maternal age. There were 4 $(14.8 \%)$ mortality. There was no major gender difference observed in development of TTS in COVID-19 unlike COVID-19 per se. Older median age group for TTS in COVID-19 patients irrespective of cardiovascular comorbidities and gender probably reflects age as an independent risk factor. Patients who developed TTS had higher mortality rate especially if they developed cardiogenic shock.
\end{abstract}

Keywords Takotsubo cardiomyopathy · COVID-19 - SARS-COV-2 - Broken heart syndrome · Stress cardiomyopathy · Takotsubo syndrome $\cdot$ Apical ballooning syndrome

This article is part of the Topical Collection on Covid-19

Hardik D. Desai

Hardikdesai198@yahoo.com

Kamal Sharma

drkamalsharma@unmicrc.org

Jaimini V. Patoliya

Jaiminipatoliya20@gmail.com

Dhigishaba M. Jadeja

Dhigishabajadeja@gmail.com

Dhruv Gadhiya

Dhruavgadhiya1992@gmail.com
1 Department of Cardiology, U.N. Mehta Institute of cardiology and research center, Affiliated to B.J Medical College, Ahmedabad 380016, India

2 Graduate Medical Education, Gujarat Adani Institute of Medical Sciences, Affiliated to K.S.K.V University, Bhuj 370001, Gujarat, India

3 Department of Biochemistry and Forensic sciences, Gujarat University, Ahmedabad 380009, Gujarat, India

4 Gujarat Adani institute of medical sciences, Bhuj 370001, India

5 Smt NHL Municipal Medical College, Ahmedabad, India 


\section{Introduction}

As of 25 November 2020, COVID-19 has affected $59,204,902$ people and $13,97,139$ death with CFR of $2.35 \%$ globally as per WHO Situation Report. Emerging evidence suggests that COVID-19 not only affects the respiratory system but can affect multiple organ systems [1]. Takotsubo syndrome (TTS) is a reversible cardiac condition, characterized by acute left ventricular dysfunction usually in the setting of physical or emotional stress [2]. In a study, Shi et al. reported a high burden of myocardial injury (19.7-27.8\%), contributing to significantly high mortality reported in these patients [3]. COVID-19 patients with cardiovascular injury have been reported to have a high burden of underlying cardiovascular comorbidities [3, 4]. One of the recent cohorts has reported an evidence of substantial (78\%) cardiac involvement in magnetic resonance imaging (MRI) among 100 German patients, who were diagnosed, treated, and recovered from COVID-19 illness. Compared to risk factor matched controls, COVID-19 patients had lower left ventricular ejection fraction (LVEF), higher left ventricle (LV) volume, high sensitive troponin T (hsTnT), and higher MRI markers of inflammation (T1 and T2 measures, 60\%) and enhancement at a median time of 71 days after diagnosis [5]. Current evidence suggests that fulminant cases of COVID-19 might have a cytokine storm or cytokine releasing syndrome, demonstrated by elevation of inflammatory cytokines [6]. Hyperinflammatory states can lead to acute stress and injury, characterized by elevated markers of inflammation and/or myocardial injury such as C-reactive protein, pro-calcitonin, creatine kinase, myoglobin, troponins, and Nterminal pro b-type natriuretic peptide (NT-proBNP) in these patients [6]. It has been observed that cytokine release syndrome is often accompanied by catecholamine surge [7], which can predispose to TTS in COVID-19 patients. Due to paucity of data on TTS in COVID-19, we aimed to perform a systemic review of individual case reports and case series of TTS in COVID-19 patients.

\section{Method}

\section{Search Strategy}

Online databases including PubMed, Google scholar, Web of Science, and the Cochrane Central Register of Controlled Trials were retrospectively searched until inception. Search strategy followed Preferred Reporting Items for Systematic Reviews and Meta-Analyze (PRISMA) guidelines by using $\mathrm{MeSH}$ and keywords like "Stress cardiomyopathy", "Takotsubo syndrome", Takotsubo cardiomyopathy", "Broken heart syndrome", "COVID-19", and "SARS-COV2 ". The search items were combined using Boolean operators ("OR"; "AND"). No filters including language, country of publication, and type of articles, including abstracts and posters, were applied. The references of individual case reports were sifted to find any relevant cases. The available results were downloaded into an EndNote library. The full search strategy is shown in the PRISMA diagram (Fig. 1).

\section{Study Selection}

We selected all these case reports and case series. Two authors (H.D and K.S) independently reviewed the abstracts, titles, and types of studies that meet eligibility criteria during phase 1. The disagreements were resolved by consensus with a third author (D.J). The second phase of the search included full text review of articles to enable identification of items for data extraction based on the inclusion criteria. Data from the article were curated and summarized in the form of country of origin, age, gender, clinical features on admission, $\mathrm{PMH} /$ comorbidities, complication during hospitalization, triggering events, medical intervention during hospitalization, and their outcome. Analysis and data collection were performed using Microsoft Excel software.

\section{Results}

The search yielded 27 patients (case reports $n=17$; case series $n=3$ ). The "baseline demographic, clinical and laboratory characteristics" and "clinical outcomes and medical management "of individual patient data are shown in Table 1 and Table 2, respectively, and case series in Table 3. Of all reported 27 cases, 22 patients' individual data was incompletely available on search. $55.5 \%$ of TTS $(n=15)$ patients were women and were more than 60 years of age $(n=8 ; 53.3 \%)$. Most of the reported cases were from the USA (43.7\%) [8-18], Italy (31.25\%) [19-23], and Spain (12.5\%) [24, 25], while Belgium [26] and Switzerland [27] contributed 1 case each. Among the cohort, 19 out of 26 (73.07\%) patients were discharged, and three were hospitalized due to acute respiratory distress syndrome and on extracorporeal membrane oxygenation or ongoing maternal age. There were 4 (14.81\%) deaths in TTS with COVID-19, which is higher than mortality reported in COVID-19 with pre-existing cardiovascular diseases otherwise (14.81\% vs 5.8\%) [28].

\section{Case Series}

Case series of Giustino $\mathrm{G}$ and colleagues reported 5 male patients of TTS with COVID-19 positive. Reported median age was 57 years (IQR: 39-65). The study noted that median time from symptoms onset to TTS diagnosis was 6.5 days (IQR: 1.0-8.0), with median value of troponin, $1.4 \mathrm{ng} / \mathrm{mL}$ (IQR: 0.55-12.55); CK-MB, $26.9 \mathrm{ng} / \mathrm{mL}$; BNP, $153 \mathrm{pg} / \mathrm{mL}$; CRP, $207 \mathrm{mg} / \mathrm{dL}$; and IL, 6-56 pg/mL. Ejection fraction was 
Fig. 1 PRISMA flowchart of study selection

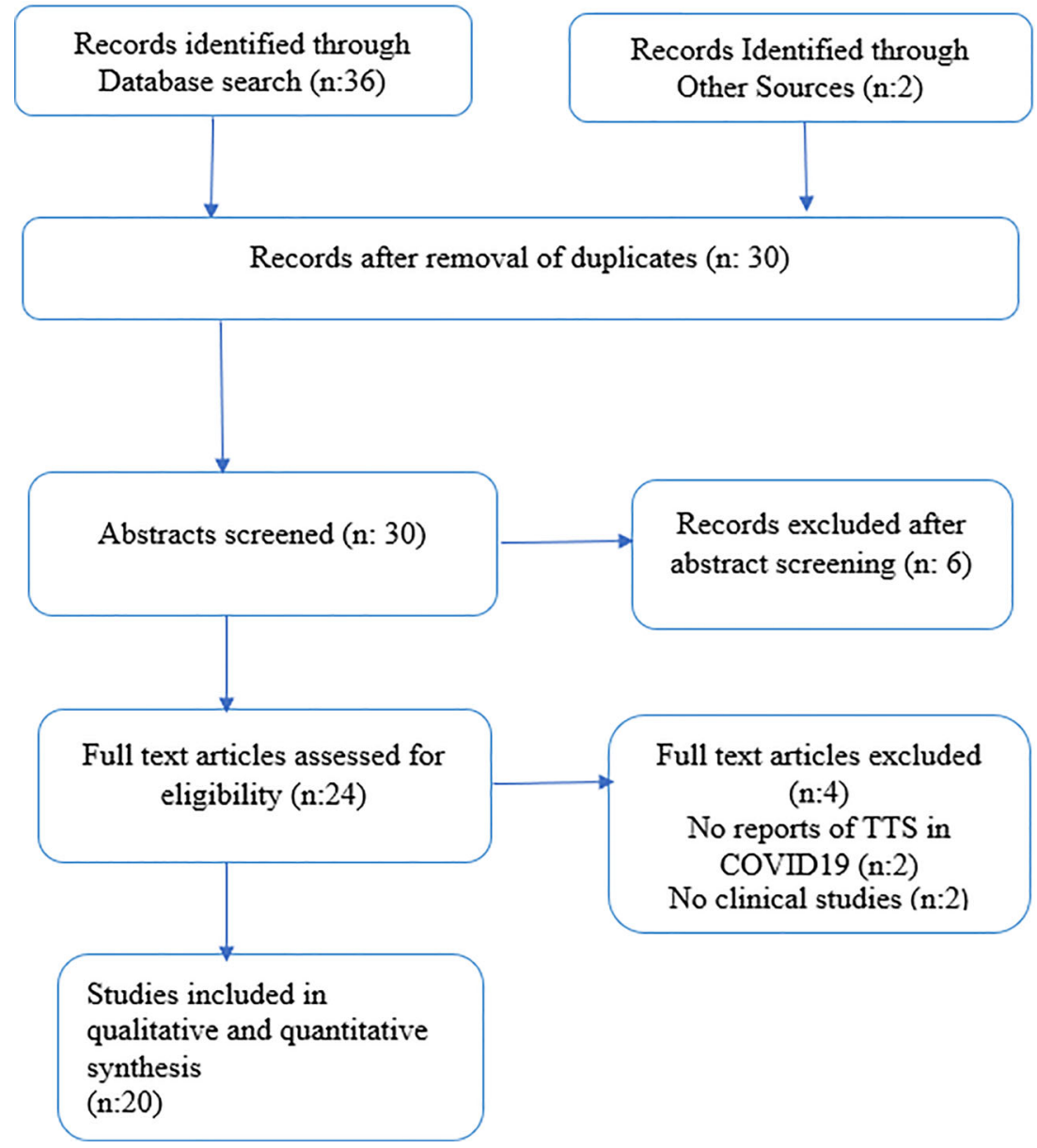

36\% (IQR: 35-37). Four out 5 (80\%) patients suffer from acute kidney injury and acute respiratory distress syndrome during hospitalization (Table 3 ).

\section{Individual Reported Cases of TTS in COVID-19}

For other 22 individual patient's data, calculated median age was 62.95 years (IQR: 50.5-73.5). The time interval from the symptom onset to TTS diagnosis was 6.7 days (IQR: 4-10). Out of these 22 cases of TTS, comorbidities reported included hypertension $(54.5 \%)$, diabetes $(36.6 \%)$, psychiatric illness $(9.1 \%)$, malignancy $(13.6 \%)$, dyslipidemia or hyperlipidemia (18.1\%), and obesity (22.7\%), and two each had ischemic cardiomyopathy and PCOD (4.54\%). Twelve out of 22 $(54.5 \%)$ patients had cardiac complications such as atrial fibrillation, pericardial effusion, cardiogenic shock, heart failure, supraventricular tachycardia, and biventricular heart failure. Twenty out of $22(90.9 \%)$ patients reported an elevated troponin $\mathrm{T} / \mathrm{I}$, reduced left ventricular ejection fraction with a median of $38.15 \%$ (IQR: $30 \%-42.5 \%$ ) [male: $40.33 \%$ (IQR: 33-44.2)] and female [37.15\% (IQR: 30-40)]. 17/22 $(77.27 \%)$ reported an elevated CRP. Eleven patients also reported an elevated D-dimer with a median of $2686.5 \mathrm{ng} / \mathrm{mL}$
(IQR: 1606-4217). 5/22 (22.72\%) reported an elevated IL-6 of mean $350.46 \mathrm{pg} / \mathrm{mL}$ (range: 67-724.38). One patient had both right and left ventricular wall motion abnormalities. All patient had specific abnormalities on ECG and cardiac imaging suggestive of TTS. Eleven out of $22(50 \%)$ patients were treated with anticoagulation, nine out of $22(40.9 \%)$ needed vasopressors during hospitalization, and seven (31.8\%) were treated with beta-blocker or diuretics. 5/22 (22.72\%) were administered tocilizumab. Nineteen out of $22(86.3 \%)$ had improvement either clinically or on repeat echocardiography from LV dysfunction.

\section{Discussion}

This is the first ever systemic review of COVID-19 patients with Takotsubo syndrome. As COVID-19 pandemic progresses globally, clear picture of cardiovascular manifestation and associated complications is emerging. Takotsubo syndrome is one of the cardiovascular complications reported in literature among COVID-19 patients. Furthermore, studies also reported increasing incidence of development of stress cardiomyopathy due to COVID-19 pandemic in COVID-19- 


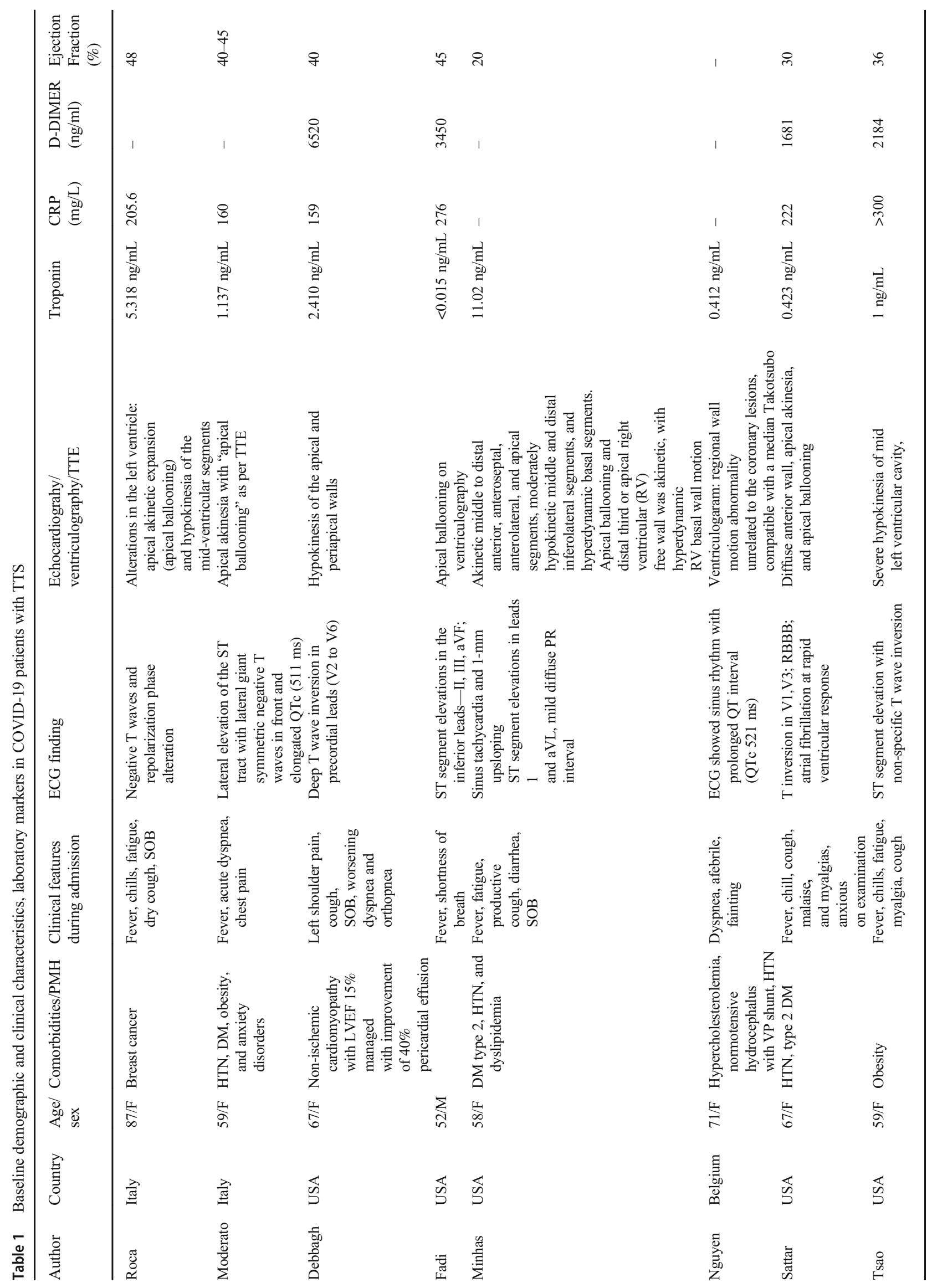




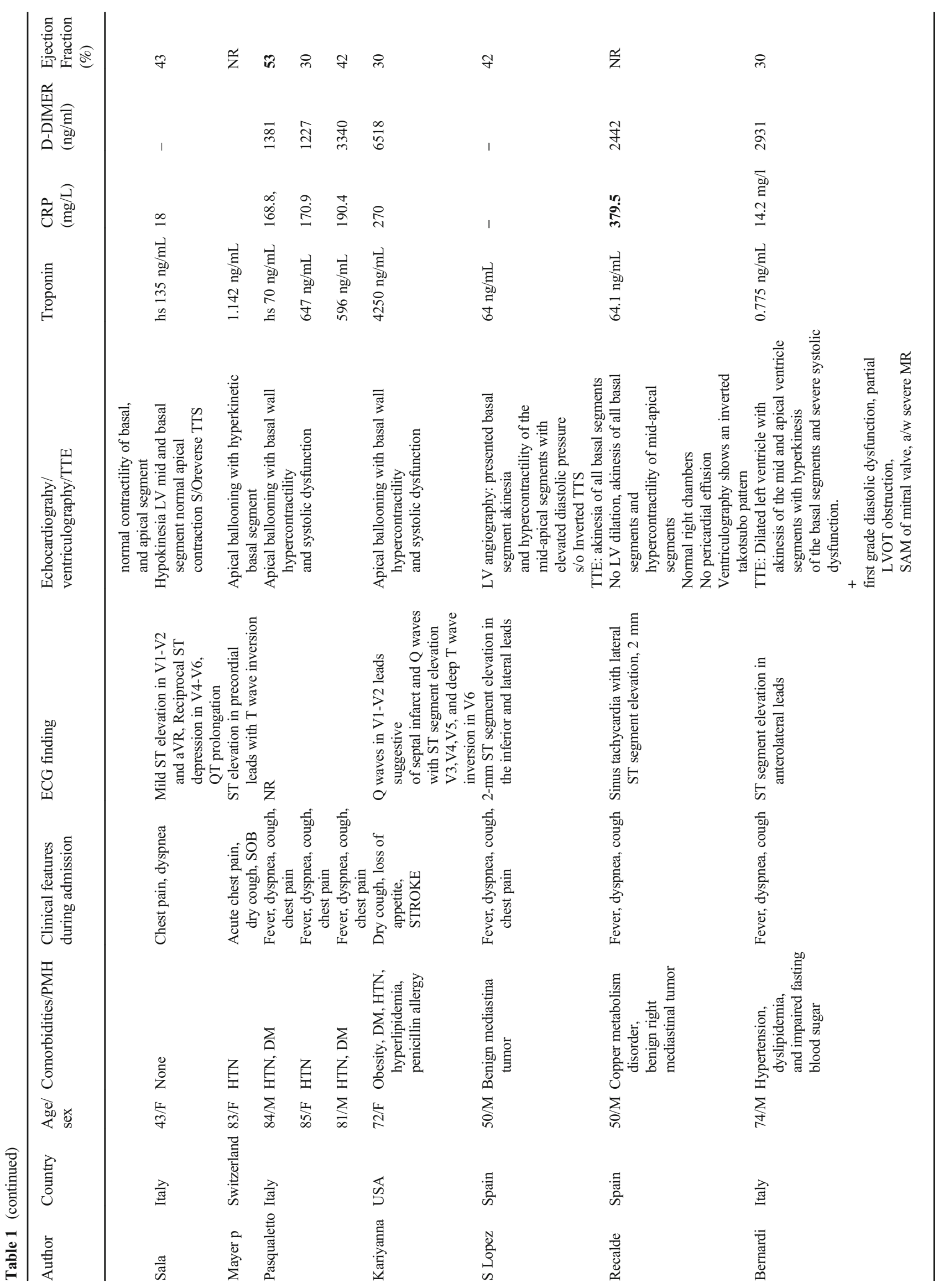




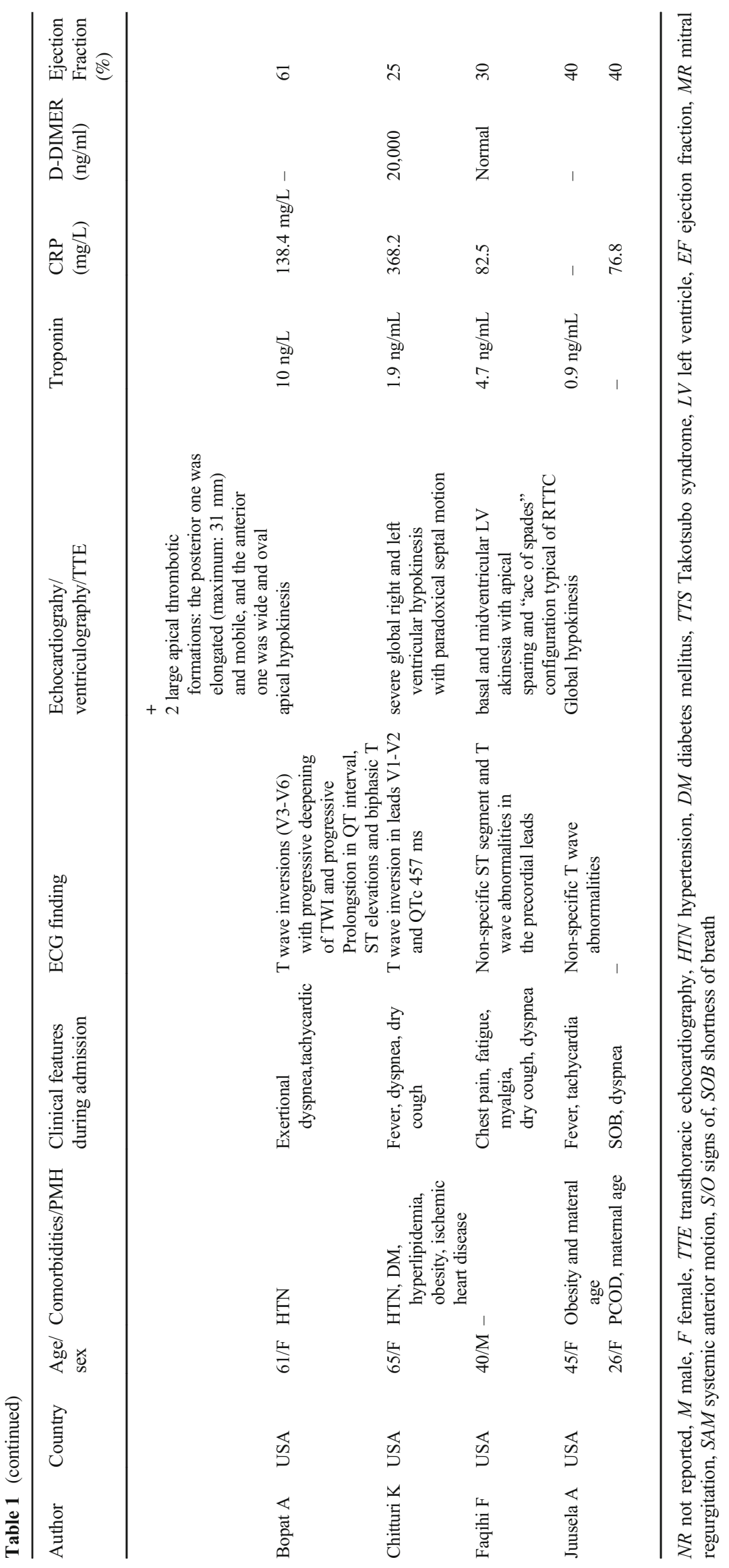




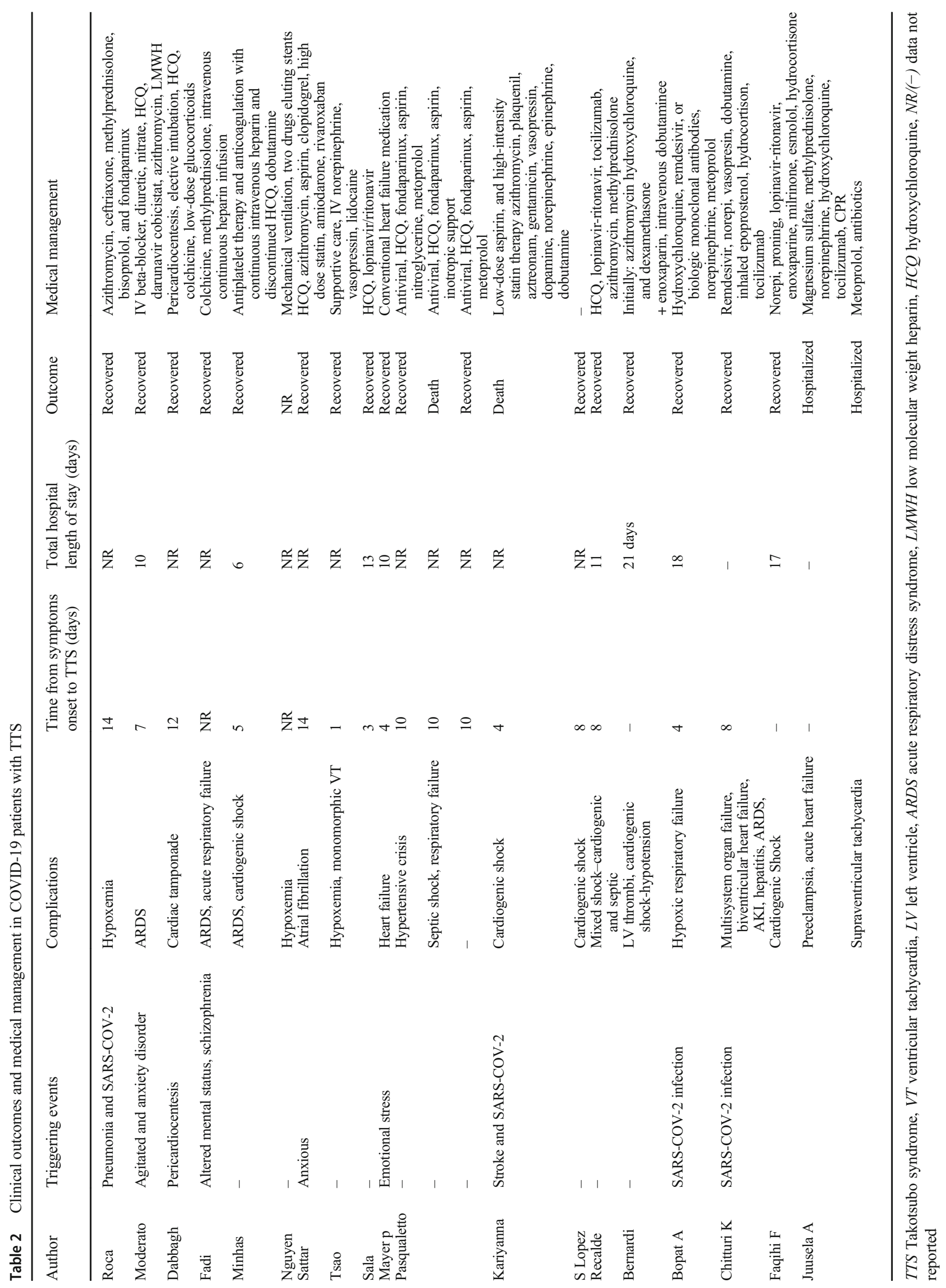


negative patients [29]. In our own previous study, we had reported male gender is equally affected by TTS with COVID-19 [30]. The current study confirms that TTS equally involves both genders in COVID-19 patients unlike TTS without COVID-19 (male $44.5 \%$ and female $55.5 \%$ vs male $10.2 \%$ and female $89.8 \%$ ) that might be due to COVID-19 affects predominantly male gender as per current evidence [2]. TTS is found to be more prevalent in postmenopausal women among females. This may be due to possible decreased levels of estrogens, as had been evident in animal models which reported that estrogen may have protective effects on cardiac myocyte by downregulation of adrenoreceptors, hypothalamo-sympathoadrenal axis, and rise in cardioprotective substance such as atrial natriuretic peptide $[31,32]$. It is hypothesized that development of TTS in COVID-19 could be due to acute stress leading to surge in the catecholamines. In this study, we have observed that various physical and emotional triggers were captured in

Table 3 Clinical and laboratory characteristics and outcome in COVID19 with TTS of case series

\begin{tabular}{ll}
\hline Variables & $\begin{array}{l}\text { Values in median } n \\
(\% \text { or IQR) }\end{array}$ \\
\hline Age, years & $57(39-65)$ \\
Male $n(\%)$ & $5(100 \%)$ \\
Clinical features & \\
Dyspnea $n(\%)$ & $4(80 \%)$ \\
Chest Pain $n(\%)$ & $1(20 \%)$ \\
Cardiac and inflammatory markers & \\
Troponin I (ng/mL) & $11.4(0.55-12.55)$ \\
D-dimer (microgram/mL) & $1.8(1.3-11.5)$ \\
CRP (mg/dL) & $207(162-277)$ \\
CK-MB (ng/mL) & $26.9(23.9-101.6)$ \\
ECG and echocardiographic finding & \\
Wall motion abnormalities $n(\%)$ & $5(100 \%)$ \\
Right ventricular dysfunction $n(\%)$ & $1(20 \%)$ \\
Pericardial effusion & $1(20 \%)$ \\
ST Segment elevation $n(\%)$ & $2(40 \%)$ \\
T wave inversion $n(\%)$ & $1(20 \%)$ \\
Sinus tachycardia $n(\%)$ & $1(20 \%)$ \\
In-hospital complication & \\
ARDS $n(\%)$ & $4(80 \%)$ \\
AKI $n(\%)$ & $4(80 \%)$ \\
Outcome & \\
Recovered and Discharged $n(\%)$ & $2(40 \%)$ \\
Hospitalized $n(\%)$ & $20 \%)$ \\
Death $n(\%)$ & \\
\hline &
\end{tabular}

$I Q R$ interquartile range, $E C G$ electrocardiography, $A R D S$ acute respiratory destress syndrome, $A K I$ acute kidney injury, $C R P C$-reactive protein, $C K-M B$ creatine-kinase $\mathrm{MB}$ development of TTS in COVID-19, such as infection due to SARS-CoV-2, psychiatric illness (schizophrenia and anxiety disorder), stroke, medical procedure (pericardiocentesis), and COVID-19 pneumonia $[8,9,13,19,20]$. Majority of patients (88.8\%) developed various complications during hospitalization such as ARDS, cardiogenic or septic shock, pericardial effusion, acute kidney injury, hypoxemia, atrial fibrillation, cardiac asystole, hypertensive crisis, and heart failure, supraventricular tachycardia, preeclampsia, and biventricular ventricular failure. Emotional factors such as fear of severity of COVID-19 itself, contact with hospitalized family member, worry about socioeconomic costs, nightmares, and intrusive thoughts of COVID-19 related to morbidity all may lead to central sympathetic hyperactivation and may contribute in development of stress syndrome/Takotsubo syndrome.

One cohort study has found a significant increase in the incidence of TTS during the COVID-19 pandemic and reported that these patients had significantly longer median hospital stay than pre-pandemic era ( 8 days vs $4-5$ days) [33]. Conditions of acute stress leading to central sympathetic activation have been suggested as potential pathophysiological mechanisms [34]. Hypercytokinemia is a hallmark of SARS$\mathrm{CoV}-2$ as it has been reported that COVID-19 is often a state of both immunodeficiency and hyperinflammation, with the latter getting manifested by a cytokine storm [6]. The elevated cytokine levels may also be responsible for the lethal complications of COVID-19 which are significantly associated with mortality. Studies have reported that rise in pro-inflammatory cytokines such as IL-6, IL-10, TNF-a, and IFN-y are observed in TTS and are associated with increase in-hospital adverse events $(<0.001)$ and mortality $(<0.05)[35,36]$. These itself can present with an increase in catecholamine levels [37], and this response itself can induce myocardial inflammation [38]. We have observed that patients with underlying cardiovascular comorbidities such as non-ischemic cardiomyopathy, hypertension, and diabetes had an elevation of pro-inflammatory cytokines such as IL-6.

This study also reports that majority of TTS patients had an elevated CRP level, and this finding was consistent with the study done by Morel et al. [39], which suggests that inflammatory markers might be related to impairment of left ventricular function. Furthermore, significant elevation of troponin and ECG changes suggestive of myocardial injury was also noted in almost all patients except one case which had normal Troponin level.

Current evidence suggests that D-dimer level significantly increases with increasing severity of COVID-19, which correlates with thrombogenic mellieu. Battrawy et al. [40] stated that patients with elevated C-reactive protein, D-dimers, and impaired left ventricular function have increased risk of development of thromboembolism in TTS patients. One case report of TTS with COVID-19 has reported a thrombus formation in left ventricle, which was successfully managed with 
enoxaparin [23]. Study done by Zhou et al. [41] has reported that patients who were treated with heparin had lower 28-day mortality compared to those who were not. In this review, we observed that (77.2\%) patients of TTS with COVID-19 had an elevated C-reactive protein and/or D-dimer. Majority of patients were treated with anticoagulation (such as low molecular weight heparin, fondaparinux, rivaroxaban, clopidogrel). Oral anticoagulation therapy should be considered in high-risk COVID-19 patients with TTS such as older age group and those with reduced LVEF. Furthermore, ten out of 17 (58.8\%) were treated with HCQ, five out of 17 (29.4\%) with antivirals, five out of 17 (29.4\%) with vasopressors, and 4 patients $(23.5 \%)$ were treated with beta-blocker. Five patients were treated with tocilizumab (IL-6 inhibitor). We observed that all patients who were treated with tocilizumab had improvement either clinically or in laboratory findings. A larger study may aid in assessing benefits of tocilizumab in high-risk cardiac COVID-19 patients and who have a significantly elevated inflammatory markers such as CRP, IL-6.

In current study, cardiac imaging demonstrated apical ballooning in most of the cases [9, 19, 20, 22, 27], apical hypo/ akinesia with or without basal hyperkinesia $[8,10,20]$, basal or mid and basal segment hypo/akinesia [10, 19, 24], and median TTS [26] characteristic of TTS variants. Four $(14.81 \%)$ patients have reported a reverse or inverted Takotsubo syndrome on echocardiography or ventriculography $[16,21,24,25]$. One patient reported both right and left ventricular wall motion abnormalities [10]. Furthermore, Majority of the patients reported non-specific ST or T wave changes ssuch as ST segment elevation in Inferior leads (II, III, aVF) or septal leads (V1, V2) or lateral leads (1 and aVL) with or without $\mathrm{T}$ wave depression in precordial leads $\}$ followed by diffuse PR interval and QTc prolongation. A wide range of cardiovascular complication reported on electrocardiographic changes in COVID-19 patients such as acute coronary syndromes, rhythm disorders, non-specific ST-T wave changes or ischemic changes, acute pericarditis, pulmonary embolism, and left ventricular hypertrophy [42]. Therefore, focused cardiac ultrasound study and critical care echocardiography performed at bedside are effective options to screen for cardiovascular complications of COVID-19 infection.

Even though most of cases recovered and were discharged (74.1\%) successfully, ten out of 27 (37.03\%) patients developed at least one of the cardiac complication such as cardiogenic shock, atrial fibrillation, heart failure, supraventricular tachycardia, or biventricular heart failure. We observed that patients with cardiogenic shock had $33.3 \%$ (2/6) mortality. Prognosis of TTS in COVID-19 patients depends on underlying triggering events as it has been reported that physical triggers in older age group could lead to worse outcomes due to larger extent of myocardial damage, and the cohort had higher mortality rate, while those with emotional trigger had better outcomes in the cohort $[43,44]$. The prognosis of TTS in COVID-19 patients was worse with $14.8 \%$ mortality as compared to $5.8 \%$ mortality in COVID-19 patients having pre-existing cardiovascular disease without TTS. This can partially be explained by physical trigger such as COVID19/SARS-Cov-2 and underlying comorbidities [13, 28].

Our study was limited by small sample size, publication bias, missing data, and lack of generalizability in demographics of the series analyzed.

\section{Conclusion}

Though the current evidence suggests that male gender is one the risk factor in COVID-19, however, there was no major gender differences observed in development of TTS in COVID-19. This may reflect females especially postmenopausal age group having an increased risk of development of TTS, thus yielding almost similar prevalence rates of TTS in COVID-19. Older median age among TTS in COVID-19 patients irrespective of cardiovascular comorbidities and gender probably reflects age as an independent risk factor. Patients with TTS in COVID-19 have higher mortality rate, more so among those who developed cardiogenic shock. Early diagnosis and management of TTS in COVID-19 may decrease inhospital mortality and cardiovascular complication. All highrisk patients with COVID-19 should be screened for TTS, and optimal treatment may be considered with antiplatelet medication, statin, and beta-blockers if indicated.

Authors' Contribution Dr. Hardik D. Desai and Dr. Kamal Sharma have equally contributed.

Data Availability Not Applicable.

\section{Compliance with Ethical Standards}

Conflict of Interest The authors declare that they have no conflict of interest.

Ethics Approval Not applicable.

Consent to Participate Not Applicable.

Consent for Publication Not applicable.

Code Availability Not Applicable.

\section{References}

1. Spuntarelli V, Luciani M, Bentivegna E, Marini V, Falangone F, Conforti G, et al. COVID-19: is it just a lung disease? a case-based review. SN Compr Clin Med. 2020;28:1-6. https://doi.org/10. 1007/s42399-020-00418-6. 
2. Templin C, Ghadri JR, Diekmann J, Napp LC, Bataiosu DR, Jaguszewski M, et al. Clinical features and outcomes of Takotsubo (stress) cardiomyopathy. N Engl J Med. 2015;373: 929-38.

3. Shi S, Qin M, Shen B, Cai Y, Liu T, Yang F, et al. Association of cardiac injury with mortality in hospitalized patients with COVID19 in Wuhan, China. JAMA Cardiol. 2020;5:802-10. https://doi. org/10.1001/jamacardio.2020.0950.

4. Guo T, Fan Y, Chen M, Wu X, Zhang L, He T, et al. Cardiovascular implications of fatal outcomes of patients with coronavirus disease 2019 (COVID-19). JAMA Cardiol. 2020;5:811-8.

5. Puntmann VO, Carerj ML, Wieters I, et al. Outcomes of cardiovascular magnetic resonance imaging in patients recently recovered from coronavirus disease 2019 (COVID-19) [published online ahead of print, 2020 Jul 27]. JAMA Cardiol 2020;e203557. doi: https://doi.org/10.1001/jamacardio.2020.3557.

6. Mehta P, McAuley D, Brown M, Sanchez E, Tattersall R, Manson $\mathrm{J}$, et al. COVID-19: consider cytokine storm syndromes and immufnosuppression HLH across speciality collaboration, UK. Lancet. 2020;395(10229):1033-4. https://doi.org/10.1016/S01406736(20)30628-0.

7. Staedtke V, Bai R-Y, Kim K, Darvas M, Davila ML, Riggins GJ, et al. Disruption of a self-amplifying catecholamine loop reduces cytokine release syndrome. Nature. 2018;564:273-7.

8. Dabbagh M, Aurora L, D'Souza P, Weinmann A, Bhargava P, Basir M, et al. Cardiac tamponade secondary to COVID-19. JACC Case Rep. 2020;2:1326-30. https://doi.org/10.1016/j. jaccas.2020.04.009.

9. Taza F, Zulty M, Kanwal A, Grove D. Takotsubo cardiomyopathy triggered by SARS-CoV-2 infection in a critically ill patient. BMJ Case Rep. 2020;13:e236561.

10. Minhas A, Scheel P, Garibaldi B, Liu G, Horton M, et al. Takotsubo syndrome in the setting of COVID-19. JACC Case Rep. 2020;2: 1321-5. https://doi.org/10.1016/j.jaccas.2020.04.023.

11. Sattar Y, Connerney M, Ullah W, Philippou A, Slack D, McCarthy B, Kroll S, Luddington S, Maya T et al COVID-19 presenting as takotsubo cardiomyopathy complicated with atrial fibrillation IJC H\&V August 2020,100580 doi:https://doi.org/10.1016/j.ijcha. 2020.100580

12. Tsao CW, Strom JB, Chang JD, Manning WJ. COVID-19associated stress (Takotsubo) cardiomyopathy. Circ Cardiovasc Imaging. 2020;13(7):e011222. https://doi.org/10.1161/ CIRCIMAGING.120.011222.

13. Kariyanna PT, Chandrakumar HP, Jayarangaiah A, et al. Apical Takotsubo cardiomyopathy in a COVID-19 patient presenting with stroke: a case report and pathophysiologic insights. Am J Med Case Rep. 2020;8(10):350-7.

14. Bapat A, Maan A, Heist EK. Stress-induced cardiomyopathy secondary to COVID-19. Case Rep Cardiol. 2020;2020:8842150. https://doi.org/10.1155/2020/8842150.

15. Chitturi K, Tacker S, Saadi M, et al. Successful treatment of heart failure in COVID-19 induced cytokine storm with tocilizumab: a case report. Eur HF J Case Rep. https://doi.org/10.1093/ehjcr/ ytaa 188.

16. Faqihi F, Alharthy A, Alshaya R, Papanikolaou J, Kutsogiannis DJ, Brindley PG, et al. Reverse Takotsubo cardiomyopathy in fulminant COVID-19 associated with cytokine release syndrome and resolution following therapeutic plasma exchange: a case-report. BMC Cardiovasc Disord. 2020;20(1):389. https://doi.org/10.1186/ s12872-020-01665-0.

17. Juusela A, Nazir M, Gimovsky M. Two cases of coronavirus 2019related cardiomyopathy in pregnancy. Am J Obstet Gynecol MFM. 2020;2(2):100113. https://doi.org/10.1016/j.ajogmf.2020.100113.

18. Giustino G, Croft LB, Oates CP, Rahman K, Lerakis S, Reddy VY, et al. Takotsubo cardiomyopathy in males with Covid-19 [published online ahead of print, 2020 Jun 4]. J Am Coll Cardiol. 2020;76(5):628-9. https://doi.org/10.1016/j.jacc.2020.05.068.

19. Roca E, Lombardi C, Campana M, et al. Takotsubo syndrome associated with COVID-19. Eur J Case Rep Intern Med. 2020;7(5):001665. Published 2020 Apr 21. https://doi.org/10. 12890/2020_001665.

20. Moderato L, Monello A, Lazzeroni D, et al. Sindrome Takotsubo in corso di polmonite da SARS-CoV-2: una possibile complicanza cardiovascolare [Takotsubo syndrome during SARS-CoV-2 pneumonia: a possible cardiovascular complication]. G Ital Cardiol (Rome). 2020;21(6):417-20. https://doi.org/10.1714/3359.33323.

21. Sala S, Peretto G, Gramegna M, Palmisano A, Villatore A, Vignale $\mathrm{D}$, et al. Acute myocarditis presenting as a reverse Tako-Tsubo syndrome in a patient with SARS-CoV-2 respiratory infection. Eur Heart J. 2020;41(19):1861-2. https://doi.org/10.1093/ eurheartj/ehaa286.

22. Pasqualetto MC, Secco E, Nizzetto M, et al. Stress cardiomyopathy in COVID-19 disease. Eur J Case Rep Intern Med. 2020;7(6): 001718. Published 2020 May 15. https://doi.org/10.12890/2020 001718.

23. Bernardi N, Calvi E, Cimino G, Pascariello G, Nardi M, Cani D, Faggiano $\mathrm{P}$ et al COVID-19 pneumonia, takotsubo syndrome, and left ventricle thrombi heart failure and imaging JACC $202015^{\text {th }}$ July. 1359-1364 doi: https://doi.org/10.1016/j.jaccas.2020.06.008

24. Solano-López J, Sánchez-Recalde A, Zamorano JL, SARS-CoV-2, a novel virus with an unusual cardiac feature: inverted takotsubo syndrome, Eur Heart J, ehaa390, doi:https://doi.org/10.1093/ eurheartj/ehaa390

25. Sánchez-Recalde Á, Solano-López J, Miguelena-Hycka J, MartínPinacho JJ, Sanmartín M, Zamorano JL. COVID-19 and cardiogenic shock. Different cardiovascular presentations with high mortality. Rev Esp Cardiol (Engl Ed). 2020;73(8):669-672. doi:https://doi. org/10.1016/j.rec.2020.04.012

26. Nguyen D, Nguyen T, De Bels D, Castro Rodriguez J. A case of Takotsubo cardiomyopathy with COVID 19 [published online ahead of print, 2020 May 12]. Eur Heart J Cardiovasc Imaging. 2020;jeaa152. doi:https://doi.org/10.1093/ehjci/jeaa152

27. Meyer P, Degrauwe S, Van Delden C, Ghadri JR, Templin C. Typical takotsubo syndrome triggered by SARS-CoV-2 infection. Eur Heart J. 2020;41(19):1860. https://doi.org/10.1093/eurheartj/ ehaa306.

28. Mehra M, Desai S, Kuy S, Henry T, Patel A, et al. Cardiovascular disease, drug therapy, and mortality in Covid-19. N Engl J Med. 2020;382:e102. https://doi.org/10.1056/NEJMoa2007621.

29. Desai HD, Sharma K, Jadeja DM, Desai HM, Moliya P. COVID-19 pandemic induced stress cardiomyopathy: a literature review. Int $\mathbf{J}$ Cardiol Heart Vasc. 2020;31:100628. https://doi.org/10.1016/j. ijcha.2020.100628.

30. Desai HD, Jadeja DM, Sharma K. Takotsubo syndrome a rare entity in patients with COVID-19: an updated review of case-reports and case-series. IJC Heart Vasc. 2020;29:100604. https://doi.org/10. 1016/j.ijcha.2020.100604.

31. Ueyama T, Hano T, Kasamatsu K, Yamamoto K, Tsuruo Y, Nishio I Estrogen attenuates the emotional stress-induced cardiac responses in the animal model of Tako-tsubo (Ampulla) cardiomyopathy. J Cardiovasc Pharmacol. 2003; 42 Suppl 1:S117-9.

32. Ueyama T, Kasamatsu K, Hano T, Tsuruo Y, Ishikura F. Catecholamines and estrogen are involved in the pathogenesis of emotional stress-induced acute heart attack. Ann N Y Acad Sci. 2008;1148:479-85.

33. Jabri A, Kalra A, Kumar A, Alameh A, Adroja S, Bashir H, et al. Incidence of stress cardiomyopathy during the coronavirus disease 2019 pandemic. JAMA Netw Open. 2020;3(7):e2014780. https:// doi.org/10.1001/jamanetworkopen.2020.14780.

34. Wittstein IS, Thiemann DR, Lima JAC, Baughman KL, Schulman SP, Gerstenblith G, et al. Neurohumoral features of myocardial 
stunning due to sudden emotional stress. N Engl J Med. 2005;352: 539-48.

35. Scally C, Abbas H, Ahearn T, Srinivasan J, Mezincescu A, Rudd A, et al. Myocardial and systemic inflammation in acute stress-induced (Takotsubo) cardiomyopathy. Circulation. 2019;139(13):1581-92. https://doi.org/10.1161/CIRCULATIONAHA.118.037975.

36. Santoro F, Tarantino N, Ferraretti A, Ieva R, Musaico F, Guastafierro F, et al. Serum interleukin 6 and 10 levels in Takotsubo cardiomyopathy: increased admission levels may predict adverse events at follow-up. Atherosclerosis. 2016;254:28-34. https://doi.org/10.1016/j.atherosclerosis.2016.09.012.

37. Pelliccia F, Kaski JC, Crea F, Camici PG. Pathophysiology of takotsubo syndrome. Circulation. 2017;135:2426-41. https://doi. org/10.1161/CIRCULATIONAHA.116.027121.

38. Roghi A, Pedrotti P, Milazzo A, Bonacina E, Bucciarelli-Ducci C. Adrenergic myocarditis in pheochromocytoma. J Cardiovasc Magn Reson. 2011;13:4. https://doi.org/10.1186/1532-429X-13-4.

39. Morel O, Sauer F, Imperiale A, Cimarelli S, Blondet C, Jesel L, et al. Importance of inflammation and neurohumoral activation in Takotsubo cardiomyopathy. J Card Fail. 2009;15(3):206-13. https://doi.org/10.1016/j.cardfail.2008.10.031.

40. El-Battrawy I, Borggrefe M, Akin I. Takotsubo syndrome and embolic events. Heart Fail Clin. 2016;12(4):543-50. https://doi.org/ 10.1016/j.hfc.2016.06.011.
41. Zhou F, Yu T, Du R, et al. Clinical course and risk factors for mortality of adult inpatients with COVID-19 in Wuhan, China: a retrospective cohort study [published correction appears in lancet. 2020 mar 28;395(10229):1038]. Lancet. 2020;395(10229):1054 62. https://doi.org/10.1016/S0140-6736(20)30566-3.

42. Angeli F, Spanevello A, De Ponti R, Visca D, Marazzato J, Palmiotto G, et al. Electrocardiographic features of patients with COVID-19 pneumonia. Eur J Intern Med. 2020;78:101-6. https:// doi.org/10.1016/j.ejim.2020.06.015.

43. Ghadri JR, Cammann VL, Napp LC, Jurisic S, Diekmann J, Bataiosu DR, et al. Differences in the clinical profile and outcomes of typical and atypical takotsubo syndrome: data from the international Takotsubo registry. JAMA Cardiol. 2016;1:335-40.

44. Pelliccia F, Pasceri V, Patti G, Tanzilli G, Speciale G, Gaudio C, et al. Long-term prognosis and outcome predictors in Takotsubo syndrome: a systematic review and meta-regression study. JACC Heart Fail. 2019;7(2):143-54. https://doi.org/10.1016/j.jchf.2018. 10.009 .

Publisher's Note Springer Nature remains neutral with regard to jurisdictional claims in published maps and institutional affiliations. 